\title{
Use of Blockchain in Governance: A Systematic Literature Review
}

\author{
Asad Razzaq ${ }^{1}$, Muhammad Murad Khan*,2, Ramzan Talib ${ }^{3}$, Arslan Dawood Butt ${ }^{4}$, \\ Noman Hanif ${ }^{5}$, Sultan Afzal $^{6}$, Muhammad Razeen Raouf ${ }^{7}$ \\ Department of Computer Science S $^{1,2,3,5,6,7}$ \\ Department of Electrical Engineering ${ }^{4}$ \\ Government College University, Faisalabad, Pakistan
}

\begin{abstract}
Blockchain is a distributed network based ledger that is secured by the methods of cryptographic proof. It enables the creation of self-executable digital contracts i.e. smart contracts. This technology is working in collaboration with major areas of research including governance, IoT, health, banking and education. It has anticipated revolutionary ways, which helps us to overcome the problems of governance such as human error, voting, privacy of data, security and food safety. In governance, there is a need to ameliorate the services and facilities with the assistance of blockchain technology. This paper aims to explore the issues of governance which can be resolved with the assistance of Blockchain features. Furthermore this paper also provides the future work directions.
\end{abstract}

Keywords-Blockchain; governance; voting; security; privacy

\section{INTRODUCTION}

Blockchain (BC) was introduced in 2008 by Satoshi Nakamoto. In order to solve the problem of double spending. Distributed ledger that digitally recorded the transactions however these transactions are encrypted and authenticated by the consensus protocol in the blockchain [1], [2]. In the network of blockchain blocks are created and maintained by using proofof-work / proof-of-stake. Each block consists of previous block hash address that maintains the historical record in the network [3], [9], [11]. Blockchain was invented as a main technology of Bitcoin, however now its application are increased in a number of fields such as governance, IOT, education, industries and health etc [28]. Moreover blockchain is the technology that remove the centralized third party by distribution of power away from the central / third party in communication, business and even politics or law.

Governance consist of all the actions of governing undertaken by any state. Currently governance is facing many issues like the privacy of data, food safety and voting etc. These issues can be resolved with the assistance of blockchain features such as decentralization, smart contract and immutability [1], [2], [3], [4], [7], [9], [11], [16]. This paper summarize the features of blockchain to resolve the current governance issues.

The challenges of BCT in governance system (such as money laundering and irreversibility) and also determined a deep understanding of governance issues with their solutions [4], [7], [25], [15]. This paper aims to explore how blockchain technology (BCT) is utilized in governance system.

The rest of this paper is ordered as follows. Section 2 covers the literature and features of blockchain. Section 3, research methodology, research questions, and research approach is examined. In Section 4 solutions of research questions are described. Section 5 discuses about blockchain framework in governance. Section 6 is provide detailed discussion of numerous challenges of validity. Finally in Section 7 conclusion and future directions are discussed.

\section{REVIEW OF LiterATURE}

This segment grants a number of fundamental thoughts and theories from prevailing research associated with the BCT and numerous functional executions in the governance. It also marks a assessment among the prevailing literature review.

\section{A. Blockchain}

A blockchain is an entire innovative technology for construction and using ledger [11], [9], [16], [21]. Blockchain records data on decentralized node called blocks. Blockchain associations the temper resistant blocks consuming mathematical cryptography and open source software computers networks and incentive mechanism [11], [16], [3], [13]. The blockchain is a trustworthy technology as it does not demand third party verification whereas, BCT verifies the authenticity of record in the network with consensus protocol [11], [21]. Blockchain responds on the distributed system by abolishing centralized authority and execute on the base of decentralized consensus protocol [11], [9]. The blockchain is cryptographic arrival against tempering on transaction stored on blocks. It allows the facility to read the data on nodes [5], [11], [13].

The blockchain is separated into three types in respect to reading the Blockchain data.

a) Public Blockchain: Allows all nodes to access the data for reading and new data purpose. Bitcoin and lite coin are the best examples of it [11].

b) Private Blockchain: Allows those nodes that are preregistered by central authorities means only has permission to authorize. Hyper ledger and ripple are the examples of it. Satoshi Nakamoto introduced the idea in 2008 and the data structure "chain of blocks" behind the crypto currency i.e., Bitcoin [11].

c) Consortium Blockchain: In this type, blockchain is not control by single authority, whereas controlled by group of approved authorities. Consortium blockchain is additionally titled as semi-decentralized [11].

1) Features of blockchain: 
a) Security: BCT is purely a $\mathrm{P} 2 \mathrm{P}$ network technology that eliminates the middle man influence/involvement [1], [2]. The transactions are validated using the consensus mechanism and authentic transaction laid down in a block that contains time-stamp and hashes of previous block [3], [1].Transaction are continuously synchronized with nodes on network and history of transaction remains visible. This $\mathrm{P} 2 \mathrm{P}$ and consensus based nature of BCT provides the security to data [1], [2], [11].

b) Decentralization: $\mathrm{BCT}$ is a digital technology that arises in its distributed peer-to-peer nature that deviates the obligation from central command [1], [7]. It allows to avoid the gathering of power that could let a single party to take control on whole setup. It helps to get rid from intermediaries [9], [16].

c) Smart contract: A process of dissemination of digital assets among two or more parties automatically stated by the formula derived on the basis of data that is interrogated at the time of setting up the contract [1]. Smart contract is a electronic program that imposes its accomplishments on blockchain enrolled by the consensus protocol [1], [4], [7], [9]. Consensus mean if all nodes on network uphold the transaction the authentication will be done.

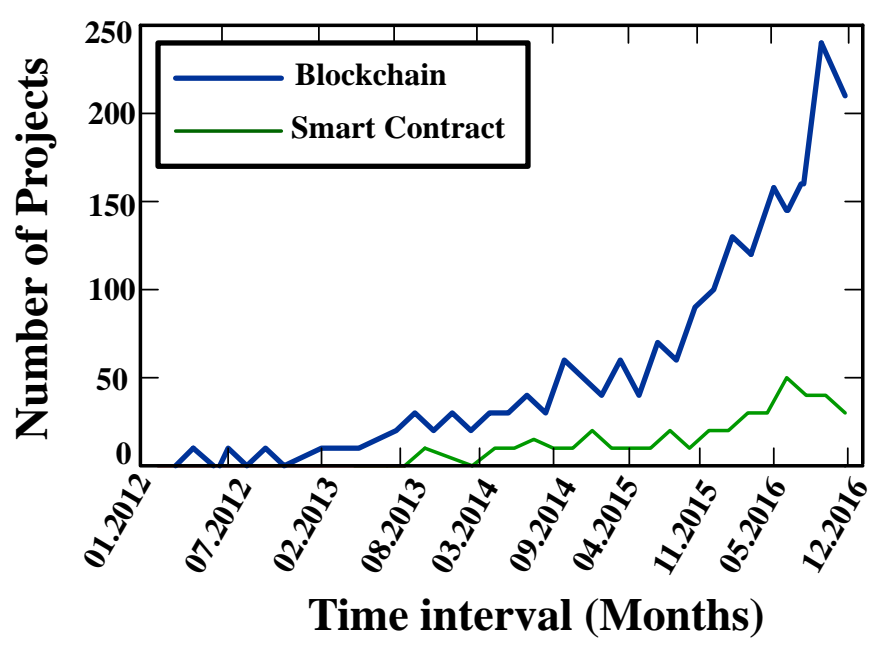

Fig. 1. Blockchain and Smart contracts increasing ratio interval of time [25].

Fig. 1 describes that with the passage of time, the blockchain projects (application) based upon smart contracts are increasing. The greatest benefit for financial engineering is "Smart contracts" computer programmable contracts that are checked and applied electronically, it creates a cheap previously agreed method to ensure that all parts of a contract are fulfilled without interference. The actual benefits of these technologies are their reliability and safety have yet to be assessed [4], [5].

d) Traceability: The traceability feature of $\mathrm{BC}$ provides the capability to figure out and track the origin of any transaction through digital signature [27].

e) Immutability: Blockchain provides a temper-proof environment in which once data has been added in block it can't be altered or removed. Immutability works totally based on 51 percent consensus involves proof of work [1].
Blockchain enables the capability to save records in very strong manners and provide the robust resistant environment to alter the data [4], [5], [6], [7], [9].

\section{B. Use of Blockchain in Governance}

Governance includes all of the procedures of governing whether undertaken by the government of a state, or by a social system (family, tribe, formal or informal organization) whether through the laws, norms, power or language of a managed society [1] It includes the mechanism needed to balance the member power (related to accountability) and the primary responsibility for enhancing the practicality of organization [20].

UK government released a report that blockchain is potential for government services. Keeping in view on this report government officially recommend the use of blockchain as a service [31].

Estonia government is using decentralized system for providing public notary facilities for their citizens. They can notarize birth certificates and educational documents etc. By using their electronic IDs provided by the state [31].

Blockchain technology can be used in voting system by government. This technology provide the anonymity of voter. The voting record always remain immutable. Danish political party used this in voting system [31].

Government apply blockchain technology for safety of food by using smart farmer market application. This application linked farmers with market, proper record about production and Transportation is grunted. This application ensure the safety and quality of food [31].

There is no hesitation in the significance of blockchain for the development and betterment of governance. Although, there always has been a struggle to find transformation technologies to support in the governance field. The area of governance is a prominent domain [19]. Blockchain governance is about determining who has the authority [12]. Blockchain technology is the amazing innovation which tries to help improve the governance field. BCT provides various benefits and used in various governance fields [13]. New technologies can reduce central administration and government costs to records, transactions, data security and system trust.

There is no cause to discard the value of governance for the progressive evolution of a country. It has been a contest to absorb assist in the governance field innovative technologies like blockchain. There has been number of creativities undertaken by the governance using block-chain technology to store the data. Dubai established blockchain based governance framework [30]. Blockchain provides benefits to Governance as payment using BCT feature smart contracts [5], [7], [17], [22]. The industry extends the concept of transactions to smart contracts. BCT features offers new ways of mutual interest and state management arranged different groups of societies in a more decentralized manner. Once two parties conduct agreement and publish it on Blockchain network that runs without human intervention [5], [7], [22]. The purpose of smart contracts is to make it easy by allowing human intervention to be taken out of the loop, thus allowing full automation [5], [9], [22]. 


\section{Compare Secondary Studies}

Since the last decade blockchain has been the favourite area of the researchers. Governance is one of the main areas of this domain. During the research secondary study could not find any regarding "Governance".

\section{RESEARCH METHODOLOGY}

A systematic literature review (SLR) means to identify, evaluate and interpret all available relevant research question [26]. To achieve this goal, The research guidelines gathered from Barbara Kitchenham which are discussed in the following subsections.

\section{A. The Aim of the Research}

Aim of this research is to present an SLR in the field of governance and highlight the issues that are being solved when works with blockchain or its application.Moreover, the issues of governance remains unsolved after BC implementation are highlighted.

\section{B. Need of Conducting the Systematic Literature Review}

The interest of this research is in the field of governance, but the review paper about area with blockchain was not found.

\section{Methodological Approach}

The tag-based methodology is to explore interrelated papers. On Google Scholar, these keywords, "Blockchain governance", "Blockchain", "Governance" and "Blockchain governance review" were explored. All the research entitlement are classified from different journals (Springer, IEEE, ACM, WILLEY and some Conference papers) that are well-balanced and downloaded after the evaluation in order to shortlist (see Fig. 2).

\section{Inclusion and Exclusion Criteria}

For inclusion, total, 52 papers were searched and shortlisted containing the required keywords "governance and blockchain". For this, the basic concept behind the primary studies was read and analysed. After analysing the abstract of papers certain studies were irrelevant to domain's concentration. Remaining research paper were really helpful and according to the required sectors. For inclusion, 32 primary studies were tagged for data extraction and others were excluded due to irrelevancy to research goal (see Fig. 2).

\section{E. Motivation and Research Questions}

The basic purpose is to present the major features of blockchain which helps to solves the issues of governance. In this modern era of technology, a revolutionary change is necessary in the governance. Therefore, the suggestions that are mandatory for the improvement of current governing system. This paper points out some issues in the governance system and resolve these issues by deep analysis of blockchain. Research questions and motivation are described in Table I.

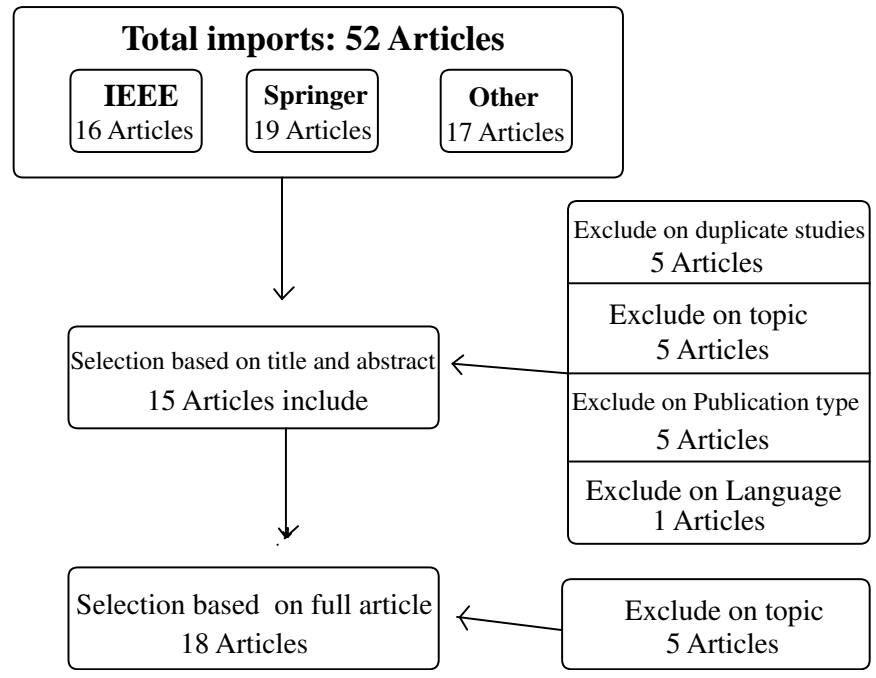

Fig. 2. Search and selection criteria.

TABLE I. RESEARCH QUESTIONS AND MOTIVATION

\begin{tabular}{c|c|c}
\hline$\#$ & Research Questions & Motivation \\
\hline \hline A & $\begin{array}{c}\text { What are the issues } \\
\text { regarding governance? }\end{array}$ & $\begin{array}{r}\text { The objective is to grab the } \\
\text { issues encountered by } \\
\text { governance structure. }\end{array}$ \\
\hline B & $\begin{array}{c}\text { What is the significant } \\
\text { feature practiced to } \\
\text { decode the problem } \\
\text { boundaries? }\end{array}$ & $\begin{array}{c}\text { Ambition to explore the } \\
\text { blockchain attribute that } \\
\text { precisely resolve the problem } \\
\text { faced by present governance } \\
\text { structure. }\end{array}$ \\
\hline & $\begin{array}{c}\text { What are unattended } \\
\text { problems? }\end{array}$ & $\begin{array}{c}\text { The purpose is to emphasize on } \\
\text { unfold those issues that cloud be } \\
\text { secured in future. }\end{array}$ \\
\hline
\end{tabular}

\section{F. Classification Criteria}

In this section, the classification of the shortlisted primary studies is presented. Shortlisted studies were classified according to research questions defined in Table II.

\section{G. Extraction of Research Data}

After delimitation of the studies related to SLR, data extraction process was started. This segment focus on derivation on most relevant data from primary resources that encountered the issues of contemporary governance system and explore features of Blockchain for reformation. The following criteria are used to extract the data from these studies:

- Issues of the Governance

- Features of BC that resolve these issues

- Unaddressed issues

\section{H. Research Historical Background}

Research in blockchain was started with the invention of Bitcoin in 2008. However, the very first paper of BCT was 
TABLE II. Classification AND SUmmary of Collected Papers

\begin{tabular}{c|c|c}
\hline \multicolumn{3}{|c}{ A. Classification } \\
\hline$\#$ & $\begin{array}{c}\text { No. of primary } \\
\text { studies }\end{array}$ & $\begin{array}{c}\text { Classification } \\
\text { criteria }\end{array}$ \\
\hline \hline A & 10 & Issues in Governance \\
\hline B & 15 & Blockchain Features \\
\hline C & 4 & $\begin{array}{c}\text { Blockchain } \\
\text { implementation issues }\end{array}$ \\
\hline
\end{tabular}

\begin{tabular}{c|c|c}
\hline \multicolumn{3}{c}{ B. Summary of Collected Papers } \\
\hline Issues & Features & Unaddressed issues \\
\hline \hline$[1][2][3][5]$ & {$[1][2][3][4][5][6]$} & \\
{$[7][10][11]$} & {$[8][9][11][12][14]$} & {$[4][7][15][25]$} \\
{$[16][22][23]$} & {$[16][18][20][22]$} & \\
\hline
\end{tabular}

published in 2015. As it's not been so long so the publication year was not considered and all the previous work related to the topic was accumulated. Research papers found in year 2016, year 2017 and year 2018 (until March) were 8, 10 and 5, respectively.

\section{RESULTS}

This section provides an explanation of the research questions that are discussed in three subcategory. The first subcategory classify the common problematic sectors of the governance structure. The second subcategory pinpoint the Blockchain features that settle down the issues of temporary Governance structure and final subcategory highlights the unattended sectors of problem area that could be secured in future.

\section{A. What are the Issues Regarding the Governance?}

1) Privacy: In this digital era, the headache of privacy of governmental records still exists due to central server storage [1], [2], [3], [11]. Data privacy is related to how information is being collected and handled.

2) Incomplete contracts: The incompleteness of a contract between authority and contractor due to an unforeseen component which may lead the government towards the problem of writing and enforcing contract cost. [11], [22].

3) Voting: It is the process of selecting aspirant in election. The traditional voting mechanism for casting the vote in designated polling station requires the handsome amount of time and money [16]. Existing voting structure enclosed with a serious flaw known as centralized design. In the existing voting system, the bogus vote can be cast that creates disaster or defragmentation in the state [3], [7].
4) Human Error: The human involvement in traditional government system plays a key role to manage the physical and digital records. However, the chance of mistake remains where a human being will manage the records manually [14], [29].

5) Food Safety: Food Safety is a problem where fresh and healthy agricultural products are not provided to the public [1]. Network becomes complex due to centralized parties like distributors, retailers, transporters and suppliers are stand between producers and end-users [10], [16].

\section{B. What is the Significant Feature Practice to Decode the Problem Boundaries?}

Table III Highlighting the one by one features of blockchain which are resolving the identified issues of governance.

1) Security: BCT is purely a P2P network technology that eliminates the middle man influence/involvement [1], [2]. Transaction are continuously synchronized with nodes on network and history of transaction remains visible. This P2P and consensus based nature of BCT provides the security to data [1], [2], [11]. This feature can overcome the following issues.

a) Privacy: Blockchain stores the transactions in network based distributed nodes so central server issue can be eliminated by shifting the data on the blockchain [1], [2]. Blockchain generates the consensus-based tempered proof transactions history that protect the sensitive data exchange on the network [3].

b) Incomplete Contract: Instead of trusted third party performing the transactions through their servers with permission, A p2p computer network running the blockchain protocol to checks the transaction by consensus [11], [22].

2) Decentralized: BCT is a digital technology that arises in its distributed peer-to-peer nature that deviates the obligation from central command [1], [7].

a) Privacy: As nature of blockchain the nodes stored the data in decentralized manner. The threat of data privacy can be minimized by shifting government institutional data on blockchain [16].

b) Voting: Due to the influence of third party in existing voting system bogus vote can be cast, decentralization feature can remove the third party so that it can reduce the issue of bogus vote [3], [7].

c) Food Safety: As decentralization remove the existence of intermediaries like distributors, retailers, transporters and suppliers. It may help the end-user to get the product directly from producer [10], [16].

3) Traceability: The feature of $\mathrm{BC}$ provides the capability to figure out and track the origin of any transaction through digital signature [27].

a) Food Safety: With the assistance of traceability feature agriculture products can be tracked digitally form ecosystem (How, when and where product was produced) to end-user [8], [9], [18]. 
(IJACSA) International Journal of Advanced Computer Science and Applications,

TABLE III. ISSUES VS FEATURES

\begin{tabular}{c|c|c|l|l|l}
\hline Issues & \multicolumn{4}{|c}{ Blockchain features } \\
\hline \hline & Security & Decentralization & Immutability & Smart contracts & Traceability \\
\hline Privacy & {$[1][2][3][11]$} & {$[16]$} & & & \\
\hline Incomplete contracts & {$[11][22]$} & & & {$[11][22]$} & \\
\hline Voting & & {$[3][7]$} & & & {$[16]$} \\
\hline Human Error & & & {$[1][4][14][29]$} & & \\
\hline Food Safety & & {$[10][16]$} & & {$[1][10][16]$} & {$[18][8][9]$} \\
\hline
\end{tabular}

b) Voting: Using traceability only the legal voters can enroll there self through digital signature and their identity can be tracked and it eliminate the threat of bogus vote [16].

4) Immutability: Blockchain provides a temper-proof environment in which once data has been added in block it can't be altered or removed [1], [4].

a) Human Error: Transaction is checked by all the nodes in consensus mechanism and this mechanism remove the probability of human error [1], [4], [14], [29].

5) Smart contracts: Smart contract is a electronic program that imposes its accomplishment on blockchain enrolled by the consensus protocol [1], [4], [7], [9]. Following issues can be resolved through smart contract.

a) Incomplete Contract: The Self- executable program helps the contract to not remain incomplete and it bounds the parties to complete the deal otherwise certain payment or transfer of digital asset not be made [11], [22].

b) Food Safety: The automation of the smart contract between the product provider and end-user eliminates the intermediary and helps in reducing cost to verify the product quality [1], [10], [16].

\section{What are Unattended Problems?}

1) Money laundering: Concealing the origin of illegally obtained money, usually through transfers, involving foreign banks or legal enterprises is known as money laundering as shown in the Fig. 3. Money laundering (ML) is a global problem. Bitcoin virtual currency provides a place for persons who generate, transfer, launder and steal illegal funds with some kind of anonymity [24]. Bitcoin offers many of the same tasks that are associated with other virtual currencies, such as Web Money, and added unique challenges to researchers because of its decentralized nature, even though there is no central Bitcoin server to compromise [25]. Possible Solution of laundering: Fig. 3 is providing two kinds of solution which has betterment or to overcome challenges that crypto coins presently pose for the global anti-money laundering (AML) [25].

a) Educate Anti money laundering task force: Firstly, in spite of some shortcomings, the risk assessment approach of the Financial Action Task Force (FATF) provides an effective balance between existing threats and opportunities that currently represent the Crypto-currency. The FATF is an officially intergovernmental organization. FATF Guidance: Three specific critics can be applied within the framework of the FATF approach, based on risk assessment. First, the FATF perpetuates greater dependency on global AML management in the market. Second according to the FATF management tends to rely on solutions based on the magic pool of Technology accordingly, Programming Application interface (APIs) provide client identification information "or third party digital identification systems that need to be adjusted themselves [25], [23].

b) Totally banned of crypto-currencies: The virtual nature of crypto-currencies (CCs), users evaluates the currency for many of the same reasons they believe that they can exchange currency-based goods, services or national currency at the date of the lateral [24]. As such, Bitcoins is currently accepted as a form of digital payment method by hundreds of legitimate retailers including vendors selling clothing, games, music, and some hotels and restaurants. If we totally banned crypto currency, money laundering will be stopped completely, but it is not possible because money gram and west union currency are used blockchain technology using virtual currency [25], [23].

2) Irreversible: Undoubtedly, the blockchain possesses remarkable properties like a distributed block, such as efficiency, irreversibility, and transparency [7]. However some disadvantages associated with this feature of blockchain [4]. In this mechanism, If data is entered incorrectly, then it can't erase/edit, because blockchain is peer-to-peer network have not reversible or erasable. So, it is a crucial issue of blockchain technology [15]. While the current settlement systems allow the process of voiding and reconciliation, imagine the problems of "rollback" in the world of chains [7].

\section{CASe Study: Dubai BlockChain Based GOVERNANCE FRAMEWORK}

Dubai established Blockchain Global Council to enhance the position of UAE as a leading centre for innovation. Dubai takes the initiative in 2000 for innovation by using blockchain. This initiative involves numerous government departments with many online services being led by Dubai Smart 

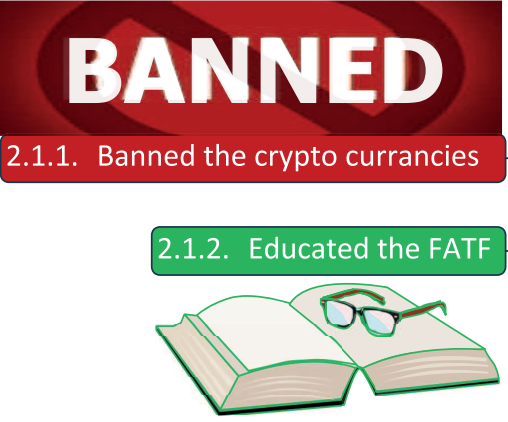



Cryptocurrancy



Fig. 3. (Money laundering solution structure using blockchain)

Government (DSG). Dubai government had set up a Dubai Vision in 2013 and build a committee which was consist of many departments involved in the vision of Smart Dubai. An executive committee for Dubai Strategy was formed in 2014 consisting governments representatives and private investors.

Finally, in 2015 blockchain based framework and DSG was approved legally for Smart Dubai. "BitOasis" and "Dubai Wills" were two pilot projects initiated by Dubai Global Counsel to enable the clients inheriting their assets and to register their wills using BCT respectively [30].

\section{Threats to Validity}

Blockchain is not hot trending area of research yet. A very small number of researches have been extracted for the support of this research till to date but this area is being favourite with the time span. Now a days many contemporary researchers are doing their explanation in concerned subject. Due to novelty of research work in BCT this research could not meet handsome number of reviews to support this research.

Due to lack of literature review it might be possible that any type of work regarding this area will be proposed by any other researcher between the closing time of this research until publication. That is being a mighty threat to this research.

\section{CONCLUSION}

The main purpose of this research is to ameliorate the government services with the assistance of blockchain. This paper is intended to highlight the problems of governance such as security, privacy, food safety and incomplete contract. The characteristics of blockchain e.g. decentralization, transparency, and smart contracts are also discussed to overcome the issues of governance. Moreover the issues of governance which are not settled yet with the assistance of blockchain such as money laundering and irreversibility. The solution is also suggested for money laundering. It will be removed by applying the proper rules and regulations as well as blockchain technology implemented on governance.

There is a need of future work on quality standards, available tools for resource allotment and determining legal framework of blockchain implementation on governance.

\section{REFERENCES}

[1] SØlnes, J Ubacht, M Janssen, "Blockchain in government: Benefits and implications of distributed ledger technology for information sharing," 2017, Elsevier.

[2] Zyskind, Guy, and Oz Nathan. "Decentralizing privacy: Using blockchain to protect personal data." In Security and Privacy Workshops (SPW), 2015 IEEE, pp. 180-184. IEEE, 2015.

[3] Noizat, Pierre. "Blockchain electronic vote." In Handbook of digital currency, pp 453-461, 2015, Elsevier.

[4] Atzori, Marcella. "Blockchain technology and decentralized governance: Is the state still necessary?" 2015.

[5] Shermin, Voshmgir. "Disrupting governance with blockchains and smart contracts." Strategic Change 26, no. 5 (2017): 499-509.

[6] Hou, Heng. "The application of blockchain technology in E-government in China." In Computer Communication and Networks (ICCCN), 2017 26th International Conference on, pp. 1-4. IEEE, 2017.

[7] Ojo, Adegboyega, and Samuel Adebayo. "Blockchain as a Next Generation Government Information Infrastructure: A Review of Initiatives in D5 Countries." In Government 3.0-Next Generation Government Technology Infrastructure and Services, pp. 283-298. Springer, Cham, 2017.

[8] Hoggett, R. D. "People, demand, and governance in future energy systems." (2017).

[9] Qi, Renming, Chen Feng, Zheng Liu, and Nezih Mrad. "BlockchainPowered Internet of Things, E-Governance and E-Democracy." In EDemocracy for Smart Cities, pp. 509-520. Springer, Singapore, 2017.

[10] Yermack, David. "Corporate governance and blockchains." Review of Finance 21, no. 1 (2017): 7-31.

[11] Davidson, Sinclair, Primavera De Filippi, and Jason Potts. "Disrupting governance: The new institutional economics of distributed ledger technology," 2016.

[12] Wang, Sha, Jean-Philippe JP Vergne, and Ying-Ying Hsieh. "The internal and external governance of blockchain-based organizations: Evidence from cryptocurrencies." In Bitcoin and Beyond, pp. 48-68. Routledge, 2017.

[13] Millard, Jeremy. "European Strategies for e-Governance to 2020 and Beyond." In Government 3.0-Next Generation Government Technology Infrastructure and Services, pp. 1-25. Springer, Cham, 2017.

[14] Chohan, Usman. "The Decentralized Autonomous Organization and Governance Issues." (2017). Carter, Nic. "A Cross-Sectional Overview of Cryptoasset Governance and Implications for Investors." (2017)

[15] Wu, Yifan. "An E-voting System based on Blockchain and Ring Signature." Master. University of Birmingham (2017).

[16] Böhme, Rainer, Nicolas Christin, Benjamin Edelman, and Tyler Moore. "Bitcoin: Economics, technology, and governance." Journal of Economic Perspectives 29, no. 2 (2015): 213-38.

[17] Tian, Feng. "An agri-food supply chain traceability system for China based on RFID blockchain technology." In Service Systems and Service Management (ICSSSM), 2016 13th International Conference on, pp. 1-6. IEEE, 2016. 
[18] Kim, Henry M., Marek Laskowski, and Ning Nan. "A First Step in the Co-Evolution of Blockchain and Ontologies: Towards Engineering an Ontology of Governance at the Blockchain Protocol Level." arXiv preprint arXiv:1801.02027 (2018).

[19] Singh, Munindar P., and Amit K. Chopra. "Violable Contracts and Governance for Blockchain Applications." arXiv preprint arXiv:1801.02672 (2018).

[20] Yli-Huumo, Jesse, Deokyoon Ko, Sujin Choi, Sooyong Park, and Kari Smolander. "Where is current research on blockchain technology? - a a systematic review." PloS one 11, no. 10 (2016): e0163477.

[21] Cong, Lin William, and Zhiguo He. Blockchain disruption and smart contracts. No. w24399. National Bureau of Economic Research, 2018.

[22] Gietzmann, Miles B. "Incomplete Contracts, and the make or buy decision: governance design and attainable flexibility." Accounting, Organizations and Society 21, no. 6 (1996): 611-626.

[23] Moser, Malte, Rainer Bohme, and Dominic Breuker. "An inquiry into money laundering tools in the Bitcoin ecosystem." In eCrime Researchers Summit (eCRS), 2013, pp. 1-14. IEEE, 2013.

[24] Campbell-Verduyn, Malcolm. "Bitcoin, crypto-coins, and global antimoney laundering governance." Crime, Law and Social Change 69, no. 2 (2018): 283-305.

[25] Bartoletti, Massimo, and Livio Pompianu. "An empirical analysis of smart contracts: platforms, applications, and design patterns." In Inter- national Conference on Financial Cryptography and Data Security, pp. 494-509. Springer, Cham, 2017.

[26] Khan, Muhammad Murad, Roliana Ibrahim, and Imran Ghani. "Cross domain recommender systems: a systematic literature review." ACM Computing Surveys (CSUR) 50.3 (2017): 36.

[27] Cartier, Laurent E., Saleem H. Ali, and Michael S. Krzemnicki. "Blockchain, Chain of Custody and Trace Elements: An Overview of Tracking and Traceability Opportunities in the Gem Industry." Journal of Gemmology 36, no. 3 (2018).

[28] Sultan A "Internet of Things Security Issues and Their Soluti ons With Blockchain Technology Characteristi cs: A Systematic Literature Review.” Am J Compt Sci Inform Technol Vol.6 No.3:27 (2018)

[29] Lomas, Elizabeth. "Information governance: information security and access within a UK context." Records Management Journal 20, no. 2 (2010): 182-198.

[30] Karmakar, Ashmita, and Ummer Sahib. "Smart Dubai: Accelerating innovation and leapfrogging E-democracy." In E-Democracy for Smart Cities, pp. 197-257. Springer, Singapore, 2017.

[31] Alketbi, Ahmed, Qassim Nasir, and Manar Abu Talib. "Blockchain for government services-Use cases, security benefits and challenges." In 2018 15th Learning and Technology Conference (LT), pp. 112-119. IEEE, 2018. 